\title{
Educación ambiental en el contexto universitario: una necesidad para la formación integral de los alumnos
}

\section{La educación ambiental en la formación de los estudiantes}

Marcos Raúl Vinces Centeno M. Sc. ${ }^{(1)}$

Jimmy Manuel Zambrano Acosta M. Sc. ${ }^{(2)}$

María Rosa Milán Licea Dr. $C^{(3)}$

(1) Universidad Técnica de Manabí, Ecuador

(2) Universidad Técnica de Manabí, Ecuador

${ }^{(3)}$ Ministerio de Educación Superior, Cuba

\section{Contacto: maravice@ hotmail.com}

Receptado: 25/02/2015 Aceptado: 12/04/2015

\section{Resumen}

La educación ambiental es un proceso que proporciona a los individuos investigar las cuestiones ambientales, permite participar en la solución de problemas y tomar medidas para mejorar los espacios donde se desarrollan las personas. Este trabajo que tuvo como campo investigativo a la “Universidad Técnica de Manabí” Ecuador, está encaminado a resolver el problema del bajo nivel educativo que se posee actualmente sobre el medio ambiente, su importancia e impacto en la sociedad. Se proyectó como objetivo realizar un análisis del nivel de los estudiantes sobre educación hacia el medio ambiente, para la proyección de una estrategia, mediante la cual se incorpore la dimensión ambiental en los programas docentes de las carreras de dicha universidad. Se utilizaron diferentes instrumentos como la observación de clases, encuestas y entrevistas a estudiantes, trabajadores y directivos de la institución. Se concluyó que existe un bajo dominio sobre el medio ambiente por parte de los estudiantes y la necesidad de trabajar en un marco en que debe operar a través de una estrategia educativa de esta dimensión; ya que es de gran importancia la inclusión de los aspectos concernientes a esta temática en los programas académicos para la formación integral de los estudiantes. 
Palabras claves: Medio ambiente, programas académicos, estudiantes, impacto social, recursos humanos

\section{Environmental education in the university context: a need for the integral formation of students}

\section{Abstract:}

The environmental education is a process that provides individuals to investigate environmental issues, it also permits to participate in solving problems and to take part in improving the places where people develop. This work that took as the researching field the "Universidad Técnica de Manabí", Ecuador is aimed at solving the problem of the low educational level that the students have on environment as well as its importance and impact on society. To objective was to make an analysis of the level of education of students about the environment for the projection of a strategy for incorporating the environmental dimension into the teaching programs of the university careers. Various instruments such as classroom observation, surveys and interviews with students, workers and directors of the institution were used. It was concluded that there is a low level of Knowledge on the environment by students and the need to work within a framework in which to operate through an educational dimension of this strategy; because it is very important to include the aspects concerning this subject in the academic programs for the integral formation of students.

Keywords: Environment, academic programs, students, social impact, human resources

\section{Introducción}

La Conferencia de las Naciones Unidas sobre el Medio Ambiente y el Desarrollo celebrada en Río de Janeiro (1992), consideró que la protección del medio ambiente, el desarrollo social y el desarrollo económico constituían pilares indispensables para conseguir el desarrollo sostenible de la sociedad contemporánea. 
De la misma forma la ONU (1992) en el documento preparatorio del Programa 21 sobre Educación, Capacitación y Toma de Conciencia se pronuncia por la necesidad de la educación ambiental y humanista.

En la investigación se abordó la responsabilidad ciudadana y el compromiso social como valores asociados desempeño profesional y por tanto, vinculados a la competencia del profesional, constituyen centro de atención en el proceso de formación que tiene lugar en las universidades en la actualidad, que se expresa en la necesidad de transitar de una formación tecnocrática a una formación humanista.

La política ambiental de la República del Ecuador debe basarse en la concentración de los esfuerzos en los principales problemas ambientales nacionales, sin descuidar los esfuerzos en los locales y sus prioridades, haciendo partícipes a todos los actores sobre la base de una acción coordinada, fundada en la cooperación y la corresponsabilidad que tribute a los intereses mundiales sobre educación ambiental, en la proyección de la ciencia y la tecnología en la solución de los problemas ambientales, propiciando la innovación tecnológica segura, lo que además contribuye a la competitividad internacional. "La gestión ambiental se enmarca en las políticas generales de desarrollo sustentable para la conservación del patrimonio natural y el aprovechamiento sustentable de los recursos naturales que establezca el Presidente de la República al aprobar el Plan Ambiental Ecuatoriano" (Novoa, 2013, p. 2)

Basado en la problemática de un nivel relativamente bajo de los estudiantes de la Universidad Tánica de Manabí en Ecuador en el tema del medio ambiente, el trabajo centro su atención en el análisis de la necesidad de una estrategia que propicie la inclusión de los aspectos concernientes a la educación medioambiental y sus impactos positivos en la sociedad en los programas académicos, con vistas a establecer un nivel apropiado en los estudiantes para enfrentar los retos que impone en mundo moderno sobre la transformación del panorama actual, donde se desarrollen los sentimientos, actitudes y valores necesarios para lograr la conciliación entre medio ambiente y desarrollo y así elevar la calidad de la vida. 
El trabajo de forma específica hizo referencia a lo planteado por vecino sobre el principio rector en la formación del futuro profesional para su participación activa en la sociedad y que para ello se requiere un análisis a fondo de los problemas que enfrenta este en el tema, para as\{i crear las bases en la proyección de una estrategia educativa coherente que precise, en los currículos y en la actividad extracurricular, las acciones que garanticen el logro de los objetivos propuestos. (Vecino, 2001)

\section{Materiales y métodos}

Para la realización de esta investigación y la búsqueda de los puntos de vistas abordados sobre el tema en este espacio de la de la vida social, se consultaron trabajos de diversos autores, cuyos aportes sirvieron de soporte teórico para la validación de la investigación sobre la necesidad de la elaboración de la estrategia.

Se observaron clases con el propósito de dilucidar el tratamiento del tema a través de los programas académicos propuestos en los planes de estudios de las diferentes carreras. Las encuestas y entrevistas fueron los instrumentos que se diseñaron tomando como punto de partida el tratamiento de los aspectos medioambientales y como repercuten estos en la calidad del proceso de enseñanza aprendizaje en el nivel universitario.

En la parte empírica se diseñó y aplicó el instrumento con el apoyo de la fundamentación teórica para la obtención de la información acertada al problema planteado. Este se realizó a través de encuestas y entrevistas a estudiantes, trabajadores y directivos con una secuencia amplia de las etapas planteadas de antemano con el objetivo de asegurar los fundamentos apropiados para la corroboración de un análisis objetivo obtener la validez de las conclusiones con respecto a la problemática formulada.

\section{Resultados}

Las universidades se convierten en uno de los pilares importantes para abordar los problemas ambientales, fundamentalmente en países como Ecuador, donde los avances en el tema ambiental han sido pocos, aislados, y sin mayores impactos para la sociedad. 
Al Sistema de Educación Superior de la República del Ecuador le corresponde participar, desde la formación de los profesionales universitarios y en la formación ambiental de la población, para lo cual debe definir objetivos y acciones agrupados por direcciones estratégicas de trabajo, encaminados al proceso de "ambientalización" de los planes y programas de estudio de manera que resulte posible incorporar la dimensión ambiental en todo el proceso docente educativo.

Para el desarrollo exitoso del trabajo, se tomaron varios referentes como el epistemológico, el pedagógico, el sociológico, así como la actividad investigativa y la vinculación de los estudiantes con la comunidad. Los dos últimos fueron campos de acción importantes dentro de la actividad educativa en la Universidad Técnica de Manabí, que sirvieron como parámetros para desarrollar actividades prácticas y de solución local de los problemas ambientales.

En este particular fue de gran utilidad dicha universidad como campo de desarrollo para la investigación. Este espacio ha sido escenario de referencia para desarrollar la actividad investigativa, que cuenta con herramientas que contribuyeron a mejorar el conocimiento y la práctica sobre medio ambiente y que además, favorecen al plano de la educación ambiental. Entre ellos fueron de gran importancia para corroborar los resultados el trabajo realizado, en el Jardín y la radio universitarios, así como un espacio televisivo estable: los que cotidianamente pueden aportar al trabajo docente que va dirigido a los estudiantes que promueva la interpretación correcta de los conceptos de Medio Ambiente y de Desarrollo Sostenible.

Para los resultados obtenidos fue de indudable importancia la ubicación de Universidad por su posición en un área de amplia riqueza natural del extremo occidental del Ecuador, en la provincia de Manabí. En este territorio, se destacan extensas áreas protegidas, como el Parque Nacional Machalilla, el Refugio de Vida Silvestre Marino y Costera Pacoche y el Refugio de Vida Silvestre Isla Corazón y Fragata. Todos constituyeron argumentos sólidos que justifican la necesidad de potenciar la educación ambiental en los profesionales que se inserten en la vida del entorno. 
A pesar de las potencialidades mencionadas, en las valoraciones hechas durante todo el trabajo investigativo, se reafirmó como problemática fundamental la presencia de insuficiencias relacionadas con la educación ambiental de los estudiantes que se forman en las diferentes carreras de la UTM. Entre ellas que se pueden destacar el bajo conocimiento en temas ambientales vinculados, directa o indirectamente, con la formación profesional específica durante los primeros cuatro semestres de las carreras universitarias.

En la referencia obtenida pudo constatarse que en ninguna asignatura se aborda el tema ambiental ni sus manifestaciones e influencia en la calidad de vida del hombre como eje transversal. No es, sino hasta el quinto semestre, en que aparece en el eje curricular la asignatura Ecología y Educación ambiental en un contexto aislado de actividades que podrían ejercer un mejor escenario para la educación ambiental. No se puede entonces considerar, que los estudiantes sean capaces de generar una reflexión desde su profesiones, que les permita actuar ante los problemas medio ambientales actuales y futuros, tan solo con la presencia de una asignatura en el eje curricular de las carreras, es decir, para saber qué hacer, entender cómo hacerlo y, además, tener voluntad de hacerlo. En éste contexto, las actividades de vinculación con la comunidad serían una oportunidad para dirigir los programas docentes hacia una interrelación entre universidad y comunidad, aspectos esenciales a incluir en la estrategia.

Los componentes entrevistados corroboraron las aserciones planteadas sobre el problema ya que sus valoraciones estriban en que estas insuficiencias están relacionadas con la escasa o nula instrumentación de acción en favor de la educación ambiental en su proceso de formación; la irrelevante inclusión de contenidos medioambientales en la planificación docente de las carreras de la Universidad, como aspectos prioritarios en el contexto, no sólo local sino mundial y el desconocimiento de la legislación en favor de la educación ambiental, esto sumado al cumplimiento aislado de acciones pedagógicas teóricas y prácticas, donde no se consideran como un eslabón importante el entorno curricular de la carrera y la universidad en su conjunto. 
De acuerdos a los aportes obtenidos a través de los diferentes instrumentos que la educación ambiental, en consecuencia, tiene que abordar la diversidad y complejidad del contenido ambiental, ubicándolo en el campo de las relaciones sociedad-naturaleza que toma como principio la unidad material del mundo que evidencia la necesidad de estudiar su contenido con un enfoque sistémico, interdisciplinario y holístico. (Díaz y Fleitas (2012)

Esta situación se evidencia en diferentes estudios realizados sobre percepción ambiental y en los diagnósticos sobre determinación de necesidades educativas, para las acciones de Formación Ambiental en los profesionales, realizados en numerosas investigaciones. (Santos 2002; Roque, 2003; Santana, 2003)

De manera general, se puedo confirmar que en la UTM no se desarrollan acciones de educación ambiental que promuevan a la búsqueda y aplicación de soluciones a los problemas ambientales del entorno, de manera que impliquen a los estudiantes en actividades de protección y mejora del medio donde viven.

La apreciaciones obtenidas permiten afirmar que se presenta entonces, una contradicción entre las exigencias educativas ambientales que demanda el entorno universitario y de la provincia de Manabí en general, y la insuficiente respuesta que ofrecen los planes de estudio de las carreras que se desarrollan en la UTM en materia ambiental, de modo que permitan enfrentar los problemas del territorio, y que los estudiantes y la población en general muestren una actitud consecuente y de conservación del entorno.

En este contexto, y como expresión del continuo perfeccionamiento de la gestión académica, es preciso incorporar la educación ambiental en correspondencia con la real conformación de una nueva cultura, que entiende las relaciones entre economía, naturaleza y sociedad desde una perspectiva holística e interdependiente y para ello es necesario trabajar en una estrategia que permita medir los resultados y ver como estos van cumpliendo los objetivos planteados. 
Todo el estudio realizado permitió puntualizar sobre el tema de las actividades profesionales, y su impacto ambiental, su acentuada actualidad y su vinculación con el paradigma del desarrollo sostenible no puede verse como un problema ideológico y como resulta indispensable identificarlo como una necesidad de atención particularmente urgente, pues ya hoy está comprometida la calidad de vida en muchas de las generaciones actuales y venideras. \}

Para el Sistema de las Instituciones de Educación Superior, este es un aspecto de importancia fundamental y a él se le debe prestar la mayor atención posible a partir de considerar que, como sistema académico, no puede desempeñar un papel pasivo dentro de todo el proceso, sino que, por su tradición y compromiso social, tiene la obligación de garantizar que los recursos docentes y de investigación se concentren en la búsqueda de alternativas aplicables, para formar un conocimiento científico sólido en las personas que, de una manera u otra, ya sea en el proceso de formación como en el de capacitación, transiten por las diferentes actividades que proponen las instituciones.

En este sentido, la creación de una estrategia permitiría formar un nuevo concepto de conocimiento, mucho más abarcador, que se apoye en la participación activa del educando en su propia formación y donde se exprese la identificación de los colectivos docentes en ese proceso, apoyada por la acción de entidades afines, constituyendo un sistema armónico que garantice una conducta profesional, individual y colectiva, encaminada a la preservación ambiental.

Para ello la estrategia que se diseñe de considerar la problemática desde diferentes ángulos. Desde el punto de vista teórico se acepta que, al abordar la concepción del medio ambiente, son elevadas las manifestaciones de reduccionismo conceptual, epistemológico y axiológico. En el orden conceptual, por considerarlo solo como el medio natural, sin tomar en cuenta lo social; en el aspecto epistemológico dado por el inductismo simple y la concepción mecanicista predominante en las representaciones sociales que sobre el medio ambiente tienen, entre otros, algunos estudiantes, educadores y ciudadanos en general, y en el ámbito axiológico ya que, en la formación de valores, muchos programas hacen 
énfasis en la educación de los comportamientos individuales y no se proyectan de manera tal que garantice el proceso de enseñanza aprendizaje histórico y contextualizado. (Núñez, 2003)

Se profundizó en la idea que durante el desarrollo del proceso de formación profesional, el sistema de conocimientos y habilidades debe orientarse a la puesta en práctica de los subsistemas de gestión ambiental durante y desde el ejercicio de la profesión y que tanto en el sistema de formación profesional como en el sistema de superación continua de los recursos humanos, procesos sustantivos que definen la razón de ser de las Instituciones de Educación Superior, debe abordarse la educación ambiental partiendo de una perspectiva participativa, interdisciplinaria y sistemática, que conciba la formación íntimamente vinculada a las restantes áreas de conocimientos contempladas en el plan de estudios y reciba, por demás, una atención particular.

Esto quiere decir que la educación ambiental, debe descansar en una filosofía académica que postule, primero, que este componente del proceso docente educativo es colectivo y participativo, lo que quiere decir que tiene que ser apoyado por factores individuales y colectivos, donde el estudiante se convierte en el protagonista principal y, en segundo término, el proceso se manifiesta de manera transversal dentro del plan de estudios aprobado.

\section{Discusión}

La formación ambiental se interpreta como una de las dimensiones de la educación ambiental que constituye un término abordado por Left (1994), quien la definen como un proceso de adquisición de actitudes, técnicas y conceptos para contribuir a una nueva forma de adaptación cultural a los sistemas ambientales a través del proceso pedagógico de formación profesional.

El trabajo propuesto en la tesis muestra que las investigaciones recientes han versado fundamentalmente la necesidad de interiorizar en la importancia que reviste para los futuros profesionales en conocimiento sobre el medio ambiente ya que esto conlleva a una visión más clara para 
la preservación del planeta, El concepto de formación ambiental es retomado por (Figueroa, 1995. P.4), al considerar esta formación como “un conjunto de actividades teóricas o prácticas que llevan a una más adecuada comprensión de los problemas ambientales y a un entrenamiento técnico (capacitación), para el manejo de los ecosistemas, en función de una adecuada calidad de vida”.

El trabajo sienta las bases para el tratamiento del tema en contexto ecuatoriano por lo que vale la pena recomendar la importancia del trabajo en estrategias que vinculen los estudiantes y trabajadores en general un acercamiento al tema medioambiental, estrategia donde se siente pautas para una educación en y para la sostenibilidad, esta se convierte en un elemento de la cultura general integral que responde al carácter unitario y sistémico del existir humano, expresando por esa vía el modo en que un hacer pedagógico actúa sobre la globalidad. Este concepto de educación integral presupone situaciones que exigen la implicación activa de todos los participantes, estudiantes, profesores y entidades, pues su mérito consiste en esto precisamente, en que se trata de acciones en las que se vive una experiencia única y singular. Su desarrollo presupone el carácter práctico en el que la persona forma parte de un colectivo en el que se ponen de manifiesto la solidaridad, la cooperación y el cuidado de la naturaleza.

Por los elementos apuntados en el análisis hecho, se define que el objetivo general es proyectar una estrategia para propiciar la Formación Ambiental de los profesionales que se forman en las Instituciones de Educación Superior, mediante la incorporación de esta dimensión al proceso docente-educativo con un enfoque interdisciplinario y orientado a la solución de problemas. Estos aspectos no contradicen los propuestos por (Figueroa ,1995; Núñez 2003; Fernández y Romero 2012) en investigaciones de intensiones similares. 


\section{Conclusiones:}

En la investigación realizada a través del análisis del comportamiento de tema de la educación ambiental se concluyó que existe desconocimiento sobre la educación acerca del medio ambiente en los estudiantes de la UTM, por lo que no se concientiza de la importancia actual que tiene el tratamiento del tema, se logró definir las características que deben manifestarse en una estrategia educativa en la temática, mediante la incorporación de la dimensión ambiental en los programas docentes de las carreras de las entidades universitarias desde en la sistematización de los presupuestos teóricos que la sustenten de manera que tribute al desempeño profesional del egresado.

Se concluyó además, sobre la urgencia que requiere el tratamiento del impacto ambiental, abordado desde las actividades académicas que desarrollan las Instituciones de Educación Superior, tanto las de carácter tradicional como las de nueva generación, desde la perspectiva de los procesos de formación y desarrollo de los recursos humanos, considerando que estos pueden ser elementos de control y balance en la dinámica de preservación ambiental, teniendo en cuenta las obligaciones profesionales como las convicciones personales y que para el cumplimiento de estas aspiraciones se hace necesario el diseño una estrategia al respecto, donde se planifique, ejecute y controle le adquisición de conocimiento y desarrollo de habilidades que para con el medio ambiente debe poseer el futuro profesional

\section{Bibliografía}

1. Díaz, O. y Fleitas, M (2012) Propuesta metodológica para la educación ambiental orientada al desarrollo sostenible desde la comunicación organizacional en la carrera de comunicación social en Guanajay. Universidad AGRARIA de La Habana "Fructuoso Rodríguez Pérez" Congreso Internacional Universidad 2012. La Habana. Cuba 
2. Fernández, I. y Romero, E (2012) La formación ambiental: componente de la formación general integral del estudiante universitario. Revista IPLAC. Publicación Latinoamericana de Educación. (2). Sección Artículo Científico

3. Figueroa, A. (1995) Formación ambiental. Revista Perspectivas Docentes. (17), 8

4. Left E., (1994) Ciencias Sociales y Formación Ambiental. Madrid: Editorial GEDISA

5. McPherson, M y col. (2004) La educación ambiental en la formación de docentes. La Habana: Editorial Pueblo y Educación.

6. Novoa, A. (). Desarrollo sostenible en la República del Ecuador. Recuperado el 12 de marzo de 2015 en http://www.unep.org/gc/gc23/documents/ecuador-desarrollo.pdf

7. Núñez, J. (2003) La ciencia y la tecnología como procesos sociales. La Habana, Editorial Félix Varela.

8. Roque Molina, Martha. (2003) Estrategia educativa para el desarrollo de la Cultura Ambiental de los futuros profesionales. IV Congreso Iberoamericano de Educación Ambiental, del 2 al 6 de junio 2003 La Habana, Cuba. Formato electrónico. docentes. Tesis de grado (Doctor en Ciencias Pedagógicas) Santa Clara, VC, ISP "Félix Varela Morales".

9. UNESCO - PNUMA. (1993) Educación Ambiental: principios de enseñanza y aprendizaje. España Departamento de Ciencias, Educación Técnica y Ambiental. -- (Serie de Educación Ambie 